\title{
Computing thermomechanical properties of dry homopolymers used as raw materials for formulation of biomedical hydrogels
}

Pavlo Demianenko $^{1,2}$, Benoît Minisini ${ }^{1 *}$, Gabriel Ortelli ${ }^{1,}$ Fabienne Poncin-Epaillard ${ }^{2}$, Mouad Lamrani ${ }^{1}$

* Benoît Minisini minisini@ismans.fr

1

ISMANS, Avenue Frédéric Auguste Bartholdi, 72000 Le Mans Cedex, France

2

Institut des Molécules et Matériaux du Mans, LUNAM Université, UMR Université du Maine -CNRS $\mathrm{n}^{\circ}$ 6283, Avenue Olivier Messiaen, 72085 Le Mans Cedex, France

J. Mol. Model. 22(7), doi 10.1007/s00894-016-3026-9 (2016)

\begin{abstract}
:
Different static properties have been calculated with COMPASS force field for polyacrylamide, poly(2-hydroxyethylacrylate) (HEA), poly(2-hydroxy ethylmethacrylate) (HEMA), poly(glycidyl-methacrylate) (GMA), polyethyleneglycol (PEG), and poly(2,2,2trifluoroethylmethacrylate) (TFEM). For each polymer, the calculated values were averaged on five equilibrated configurations of amorphous cell composed of one atactic chain containing 100 repeat units. The ranking obtained fromthe densities calculated at $300 \mathrm{~K}$ is TFEM $>$ HEA $\approx$ xpolycrylamide $>$ HEMA $\approx$ GMA $>$ PEG. Concerning the glass transition temperature we have obtained polyacrylamide $>$ HEMA $\approx$ GMA $\approx$ HEA $>$ PEG, and polyacrylamide $>$ HEMA $\approx \mathrm{HEA}>\mathrm{GMA} \approx \mathrm{PEG}>\mathrm{TFEM}$ for the bulk modulus. The calculated results, when available, have been compared with experimental data coming from literature.
\end{abstract}

Keywords : Dynamics simulation . Polymer-thermomechanical . Properties-molecular

\section{Introduction}

In 2004, it was estimated that 125 millions people (2\%) used contact lenses world wide [1] whereas the compound annual growth rate of the sector is expected to rise to $10.3 \%$ from 2013 through 2019 [2]. In the aim to fulfill the still rising consumers expectations, intensive research must be undertak- 
en by the leading companies. PMMA, patented for corneal contact lens, was the first polymer ever used for such application and since the mid-1950s the number of polymeric materials used for such application does not cease to increase. Currently, different polymer materials such as polyacrylamide, poly(2hydroxyethylacrylate) (HEA), poly(2-hydroxyethylmethacrylate) (HEMA), poly(glycidylmethacrylate) (GMA), polyethylene glycol (PEG) and poly(2,2,2-trifluoroethylmethacrylate) (TFEM) are frequently mixed or copolymerized in different proportions to satisfy the drastic criteria based on biocompatibility, oxygen permeability, optical clarity, and mechanical properties. In addition, chemical compatibility is another important aspect since no fewer than 26 molecular agents (cross-linker, viscosity adjuster, solubilizer, surfactant, antioxidant, anti-histamine, color indicator, disinfectant, lipid layer enhancer, moisturizer, $\mathrm{pH}$ adjuster, protein remover, osmolality adjuster, vasoconstrictor) can enter in contact lens formulation or being contact with them.

Due to this complexity, it's not rare that computer-aided materials design strategy be used in parallel to analytic or experimental works to develop new hydrogel formulation. However, the predictive character of empirical atomistic simulations relies on different parameters well summarized by van Gunsteren [3]. Among them, force field transferability, size models, and sampling are among the most important factors. United [4-7] or All [7-22] atoms force fields were extensively used during the last 20 years to study phase behavior [8,9], structures [10-12], transport [13-19], mechanical [7,20,21], and thermodynamic [22] properties of hydrogel models from Monte Carlo [8,9] or molecular dynamic simulations [7, 10-22]. Among the different available force fields, OPLS_AA was utilized to calculate the interactions in $\mathrm{N}$-isopropylacrylamide pure water [11] or ethanol-water [12] mixture and PVA [10,22] models. PVA hydrogel models were also performed with tailor-made [16], AMBER [13], COMPASS $[14,15]$ force fields. Poly(ethyleneglycoldiglycidylether) -Poly(bis(trimethylsilyloxy) methylsilylpropyl glycerol methacrylate) [7] and poly(2methacryloy-loxyethylphosphorylcholine) [18] studies were carried out by means of COMPASS whereas investigations were achieved with DREIDING on complex model of poly(N-vinyl2-pyrrolidone-co-2-hydroxyethyl methacrylate) [17] and poly(ethylene oxide)poly(acrylic acid) [20] network hydrogel.

In their review about molecular simulation of polymeric network and gel, dating from 1999, Escobedo and de Pablo [8] discussed the necessity to model large polymer networks to take into account topological irregularities. However, in spite of progress in parallelization calculation, the size of full atomistic models is still generally limited to several tens of thousands of atoms. In 2010, Walter et al. [11] studied the radius of gyration of PNIPAAm single chain of 30 monomers in 14,482 water molecules for different temperatures. This degree of polymerization, after comparison with results obtained for DP 
$=15,30,50,75$, was assessed as giving significant results with a reasonable computational effort. Three days of calculation, on 128 cores of a PC-cluster composed of HP XC 4000 equipped with Opteron 2.6GHz Dual Core processors, were required to simulate the evolution of the system during 40 ns. Consequently, in a first approach, the molecular agents are generally neglected leading to atomistic models mainly focused on the polymer architecture in water. As an example, soft contact lenses developed at the end of the 1980s [23] could contain one or more hydrophilic monomers. Currently, in silicone-hydrogel contact lenses [24], it is not rare that one or two silicone monomers were blended with at least one hydrophilic monomer. Therefore, the latest atomistic models were generally composed of thousands of atoms coming from the replication of at least three distinct molecular groups. For instance, recent studies deal with poly(bis(trimethylsilyloxy)methylsilylpropyl glycerol methacrylate) (PSiMA) and poly(2-methacryloyloxyethyl phosphorylcholine) (PMPC) interpenatring network model [18], hydrogel and silicone-hydrogel [19] or poly(N-vinyl-2pyrrolidone-co-2-hydroxyethylmethacrylate) [17] . Moreover, considering the polymeric system, the properties are independent of the molecular weight but it is not true for oligomers and generally MD simulation using all atoms force field deals with oligomers. Therefore, the calculated properties, obtained for oligomers, are difficult to compare with experimental results measured for polymers. The impact of the degree of polymerization on the solubility parameter evaluated for PVA models has been shown by Noorjahan and Choi in 2013 [22]. They observed a drop from 6, 3, and 2.5 MPa respectively for atactic, isotactic, and syndiotactic PVA when the degree of polymerization was increased from 50 to 400 monomer units.

Consequently, when interested in the prediction of the properties of multicomponent systems, it's important in a first step to assess the reliability of the properties predicted for pure components. If the predicted properties are not, at least qualitatively, comparable with the experimental ones for the pure components, good correlation with experimental results for a multicomponent system can be anything but truthfully. In the present work we will focus on the COMPASS force field which has been previously used for hydrogel study. However, to our knowledge no systematic study on pure polymers susceptible to enter in the composition of commercial hydrogel exists. That's why, we used a systematic way, based onthe method developed by Metatla and Soldera [25] to build and equilibrate pure models of five hydrophilic polymers polyacrylamide, HEA, HEMA, GMA, PEG, and one hydrophobic polymer TFEM. We will fix the degree of polymerization to 100 monomers units for the all models. Our properties of interest include density, elastic properties, solubility parameters, and glass temperature calculated from MD simulation and averaged on five configurations. Comparison was made with experimental and group contribution results when available. 


\section{Method}

Modeling and simulations were performed using different modules available in Materials Studio 5.0 software suite. For each polymer, one atactic chain containing 100 monomer units with a head to tail regiochemistry was built. Chemical details of the repeat units and the size of the modeling polymer chains are given in Table 1. We decided to work at constant degree of polymerization leading to an important difference concerning the molecular weight of the different polymer chain. The molecular weight of the heavier chain, TFEM, is thus around 13 times larger than for the lighter one, PEG, whereas the number of atoms ranges from 702 for PEG to 2102 for TFEM. Amorphous polymer models were generated using the Theodorou and Suter [26] method as implemented in the amorphous cell module. For each polymer, 50 cells containing one chain were generated in the aim to explore the configuration space. The temperature and the density were fixed at $298 \mathrm{~K}$ and 0.6 respectively whereas the COMPASS [27] force field was used to determine the energies of the added dihedral angles during the chain growth. The nonbonded terms of the potential energy were calculated using the Ewald summation method with an accuracy of $10^{-2} \mathrm{kcal} \mathrm{mol}^{-1}$. The radius of gyration was calculated for all configurations and provides us a first criterion of configuration quality. From the distribution, we selected the configurations belonging to the class with the highest probability. The second criterion was therefore based on energy. These structures were optimized during 10,000 steps and the five lowest energy configurations were selected for the relaxation process. To converge toward the simulated density we began with a simulated annealing. To keep the system at prescribed temperature $(298 \mathrm{~K})$ and pressure $(0.1 \mathrm{GPa})$ the Berendsen thermostat and barostat were employed. A simulated annealing was performed with the temperature rising from $300 \mathrm{~K}$ to $800 \mathrm{~K}$ and cooling down from $800 \mathrm{~K}$ to $300 \mathrm{~K}$ with a step of $25 \mathrm{~K}$ and a maintained time of 25 ps. To finish the equilibration process, the cells were compressed uniformly with variation steps of the cell edge and an energetic convergence of $10 \mathrm{kcal} \mathrm{mol}^{-1}$. The volume at equilibrium and the bulk modulus at $0 \mathrm{~K}$ were extracted from the fitting of the energy versus volume curve with a second order polynomial regression model. The final value was averaged on the five configurations. We considered as the equilibrated structures, the configurations of lowest energy obtained for each energy vs volume curve. From these configurations different static properties were evaluated. 


\begin{tabular}{|c|c|c|c|c|}
\hline Acronym & Formula & Name & Molecular Weight & Atom Number \\
\hline Acrilamide & & Poly(Acrylamide) & 8502 & 1002 \\
\hline HEMA & & Poly(2-Hydroxyethyl Methacrylate) & 13002 & 1902 \\
\hline HEA & & Poly(2-Hydroxyethyl Acrylate) & 11602 & 1602 \\
\hline TFEM & & Poly(2,2,2-trifluoroethyl meth acrylate) & 18202 & 2102 \\
\hline GMA & & Poly(glycidyl methacrylate) & 14202 & 2002 \\
\hline PEG & & Poly(ethylene glycol) & 1402 & 702 \\
\hline
\end{tabular}

Table1 Characteristics of the different polymers. Molecular weights are givening mol $^{-1}$

\section{Elastic constants}

The stiffness tensors were evaluated on the different configurations of lower energies. Then the different components of the tensor were averaged on the five configurations for each polymer. As an amorphous cell, the system should be isotropic, leading to only two independent elements present in the stiffness tensors.

$$
\left(\begin{array}{cccccc}
2 \mu+\lambda & \lambda & \lambda & 0 & 0 & 0 \\
\lambda & 2 \mu+\lambda & \lambda & 0 & 0 & 0 \\
\lambda & \lambda & 2 \mu+\lambda & 0 & 0 & 0 \\
0 & 0 & 0 & \mu & 0 & 0 \\
0 & 0 & 0 & 0 & \mu & 0 \\
0 & 0 & 0 & 0 & 0 & \mu
\end{array}\right)
$$

Bulk(B) and Young's (E) moduli can therefore be derived from the Lamé coefficients :

$$
B=\lambda+\frac{2}{3} \mu ; E=\frac{\mu(3 \lambda+2 \mu)}{\lambda+\mu}
$$




\section{Solubility parameter}

The solubility parameter $(\delta)$ and the cohesive energy density was calculated from each equilibrated structure by :

$$
\delta=\sqrt{C E D}=\sqrt{\frac{E_{\text {iso. }}-E_{\text {vol }}}{V_{\text {vol }}}}
$$

Where $\mathrm{E}_{\mathrm{iso}}$ is the energy of the polymer chain in the vacuum, $\mathrm{E}_{\mathrm{vol}}$ is the energy of the polymer chain in the cell of volume $\mathrm{V}_{\mathrm{vol}}$.

\section{Glass transition temperature}

The dilatometric technique was employed to determine the glass transition temperature (Tg). As the system cools down, the specific volume was reported for the different temperatures. In our case the temperature ranges from $800 \mathrm{~K}$ to $200 \mathrm{~K}$ or $100 \mathrm{~K}$. The departure from a linear relationship between the specific volume and the temperature yields the value of the $\mathrm{Tg}$. The cell at equilibrium was heated to

$800 \mathrm{~K}$ with a heating rate of $3 * 10^{13} \mathrm{Kmin}^{-1}$. There after, the system was cooling down to $200 \mathrm{~K}$ or $100 \mathrm{~K}$ by $25 \mathrm{~K}$ steps of $200 \mathrm{ps}$ duration each, giving a cooling rate of $7.5^{*} 10^{12} \mathrm{kmin}^{-1}$, these data were used to evaluate the $\mathrm{Tg}$ andthe density at $300 \mathrm{~K}$.

The different computed values were compared to values calculated from contribution group method as presented in $[13,28,29]$ and implemented in the module Synthia available in Materials Studio 5.0. This technique relies on the fact that empirical relations between the properties of interest and some functional group descriptors such as chemical composition, connectivity, valence states, ect. have been developed from a reliable polymer database. These relations are then supposed to be transferable for new monomer units as far as they can be divided into any known functional group. So the new repeat unit is first divided into known groups, then the contributions of each descriptor are retrieved from a database and the properties are calculated from empirical relations. Concerning the prediction of the solubility parameter two relations exist developed respectively by Fedors and Van Krevelen on the basis of the group contributions of the cohesive energy and the molar volume [30].

\section{Results}

The values of the different properties calculated for the six polymers are summarized in Table 2. The different results will be discussed in the following sections : density, glass transition temperature, mechanical properties, solubility parameter. 


\begin{tabular}{c|c|c|c|c|c} 
& $\begin{array}{c}\text { Density } \\
(300 \mathrm{~K})(-)\end{array}$ & $\mathrm{Tg}(\mathrm{K})$ & $\begin{array}{c}\text { Solubility } \\
\text { parameter } \\
\left(\mathrm{J}^{1 / 2} / \mathrm{m}^{3 / 2}\right)\end{array}$ & $\begin{array}{c}\text { Young } \\
\text { modulus (GPa })\end{array}$ & $\begin{array}{c}\text { Bulk modulus } \\
(\mathrm{GPa})\end{array}$ \\
\hline FEM & 1.38 & 429 & 12 & 5.3 & 5.3 \\
\hline HEMA & 1.21 & 403 & 20.6 & 8.7 & 9.2 \\
\hline Acrylamide & 1.32 & 395 & 28 & 12.8 & 11.2 \\
\hline GMA & 1.2 & 461 & 14.3 & 6.7 & 7.6 \\
\hline HEA & 1.27 & 411 & 18.5 & 8.9 & 9.2 \\
\hline PEG & 1.14 & 285 & 19.4 & 6.5 & 8.6
\end{tabular}

Table2 Summary of properties calculated for the investigated polymers by molecular dynamic simulation

\section{Density}

A comparison of the computed densities evaluated at $0 \mathrm{~K}$ and $300 \mathrm{~K}$ with literature and group contribution values at $300 \mathrm{~K}$ is presented in Fig. 1. For the data at $0 \mathrm{~K}$, the values were obtained from the fitting of the five energy versus volume curves, presented in Fig. 2, with a second order polynomial regression model $\left(\mathrm{E}(\mathrm{V})=\mathrm{aV}^{2}+\mathrm{bV}+\mathrm{c}\right)$. This approximation holds as long as the fitting points are chosen close to the minimum of the curve. Five points, including the minimum, were taken to evaluate the three parameters. The volume at equilibrium (Vmin) is evaluated from $\mathrm{Vmin}=-\mathrm{b} / 2 \mathrm{a}$. The error bar in this case only takes into account the standard deviation of results obtained on our five configurations. For the results at $300 \mathrm{~K}$, the standard deviations take into account both temporal and configurational variations.

From the data of Gary et al. obtained for polystyrene, and discussed by Ding et al. [31], it appears that the density of oligomers $\left(\mathrm{Mw}=1 \mathrm{~kg} \mathrm{~mol}^{-1}\right)$ is around $95 \%$ lower than for polymer $\left(\mathrm{Mw}=100 \mathrm{~kg} \mathrm{~mol}^{-1}\right)$. This is observed for TFEM and HEA, with calculated values lower by less than5\% in comparison to the experimental data. However, they are higher by less than $6 \%$ for polyacrylamide, PEG and HEMA. The highest discrepancy is for GMA with a value of $12 \%$ as the reference density is assumed to be the same as for poly(propyl methacrylate), an assumption recently made by Zdyrko et al.[32]. The disagreement is even more important if we compare with the value of 0.805 given at $25{ }^{\circ} \mathrm{C}$ by a Sigma Aldrich for a polymer with a $\mathrm{Mn} \approx 10-20 \mathrm{~kg} \mathrm{~mol}^{-1}$. However, 0.805 appears extremely low in comparison to 1.07 the density given for the monomer by the same vendor. Different explanations can be proposed to clarify these discrepancies. It's well known that the density of polymers is highly influenced by the temperature, the tacticity, the water content or the morphology. These characteristics were not always given in the literature, especially the tacticity and the water content. The presence of the epoxy groups in GMA is another point of concern since no information is given concerning their reactivity. In our simulation we considered that the epoxy group remained unreacted. From the literature data, the densities increase in the order of TFEM $>$ HEA $\approx$ polyacrylamide $>$ HEMA $\approx$ PEG $>$ GMA. Concerning the calculated data, as 
expected the densities at $0 \mathrm{~K}$ were higher than those at $300 \mathrm{~K}$ but ranking is similar for the two temperatures TFEM > polyacrylamide > HEMA > HEA $\approx$ GMA > PEG. The densities evaluated from group contribution method [29], were situated between the calculated densities obtained at $0 \mathrm{~K}$ and $300 \mathrm{~K}$ for HEA and HEMA ; whereas, these values were lower than the calculated densities by force field method polyacrylamide and PEG and higher TFEM.

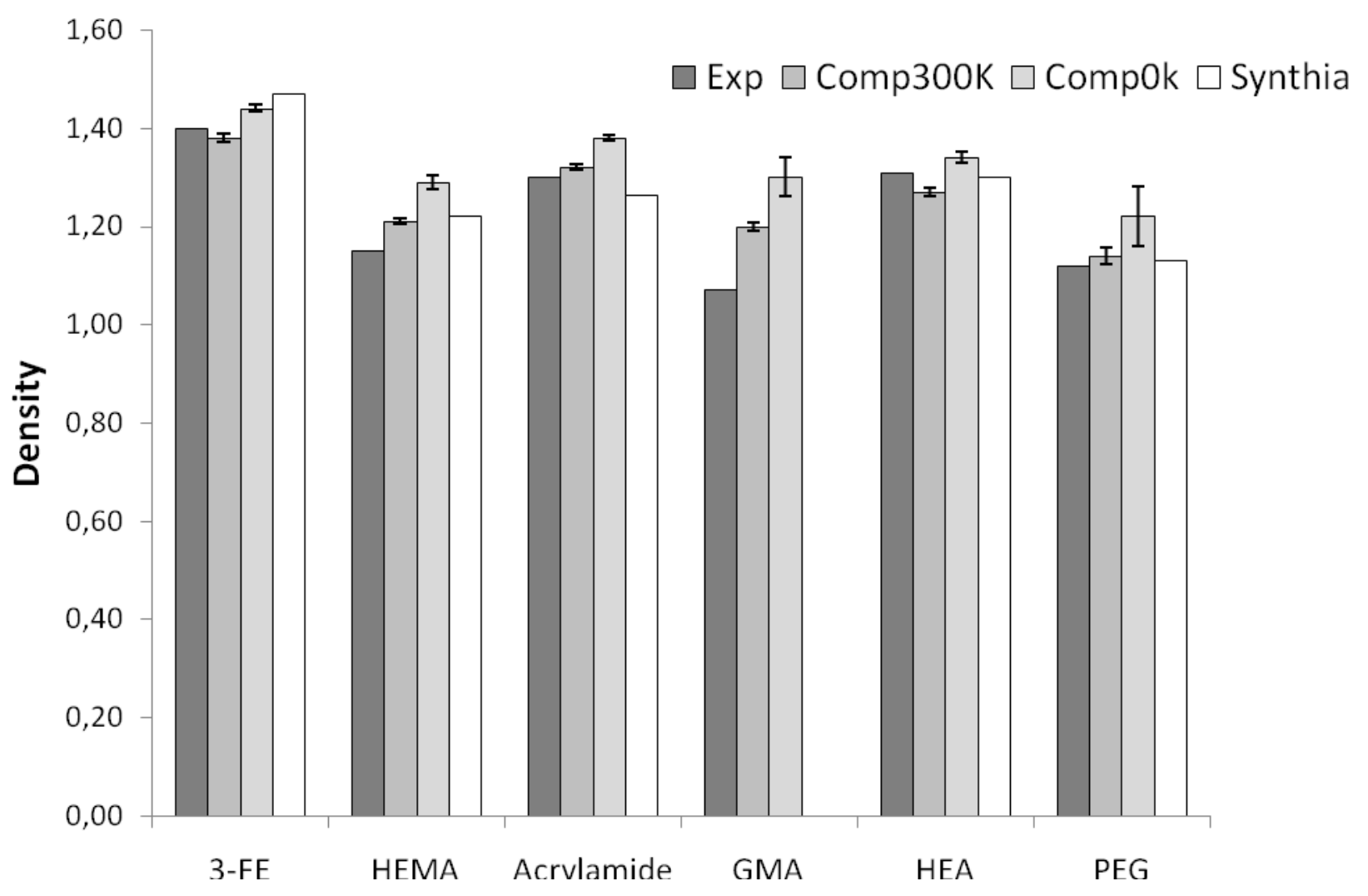

Fig.1 Comparison of calculated densities at 300K by molecular dynamic (MD) and 0K by molecular mechanic (MM) with experimental results (http://www. sigmaaldrich.com/) and group contribution method (Gr.Contr.) 

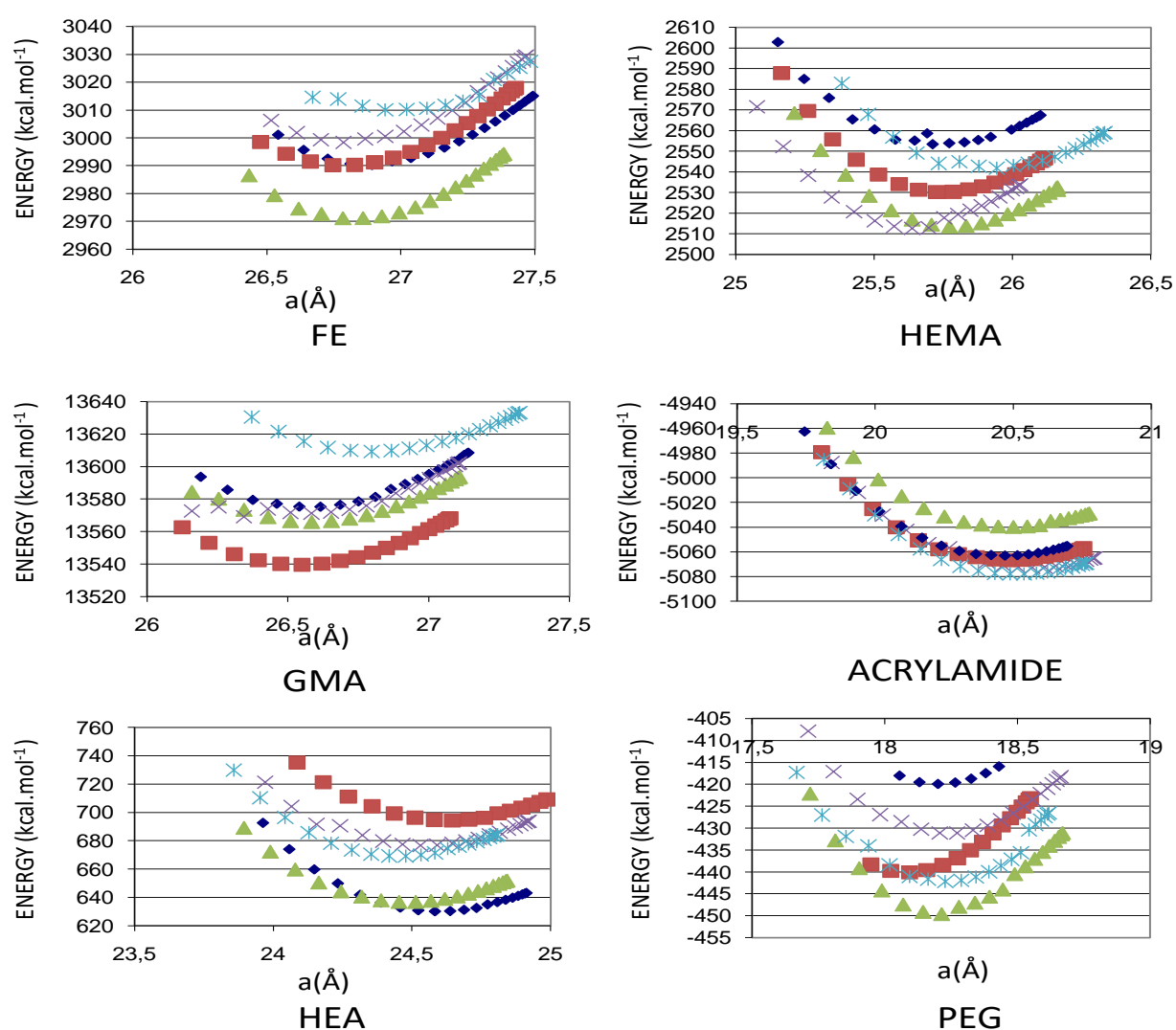

Fig.2 Curves of energy with respect to the amorphous cell volume during a uniform static compression for five configurations

As a conclusion, and before getting more accurate experimental data for dry oligomers, our calculated densities seem in correct agreement with the literature data.

\section{Glass transition temperature}

Specific volume versus temperatures curves for all polymers are given in Fig. 3. Linear regression was used to fit the data in the glassy area. The interception with the y axis allows us to extract the density at $0 \mathrm{~K}$ from a different way than previously done from energy versus temperature curve. The extrapolated density at $0 \mathrm{~K}$ are 1.25, 1.28, 1.29, 1.34, 1.36, 1.48 for PEG, GMA, HEMA, HEA, poly(acrylamide), and TFEM respectively. These results are consistent with those exposed in Fig. 1 since the highest discrepancy is less than $4 \%$ for TFEM. The slopes give us the coefficient of thermal expansion. In the glassy region the values are $1.06,1.49,1.79,1.85,2.02,2.09 * 10^{-4} \mathrm{~K}^{-1}$ for HEMA, HEA, TFEM, polyacrylamide, GMA, PEG respectively. 


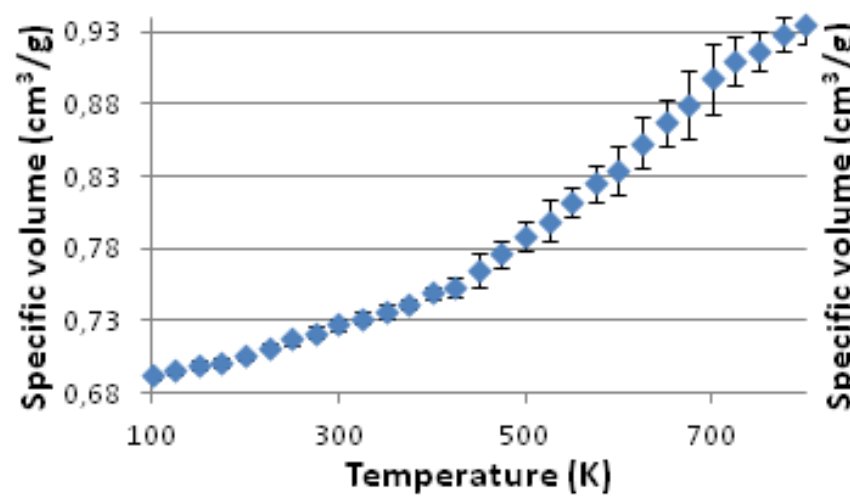

3-FE
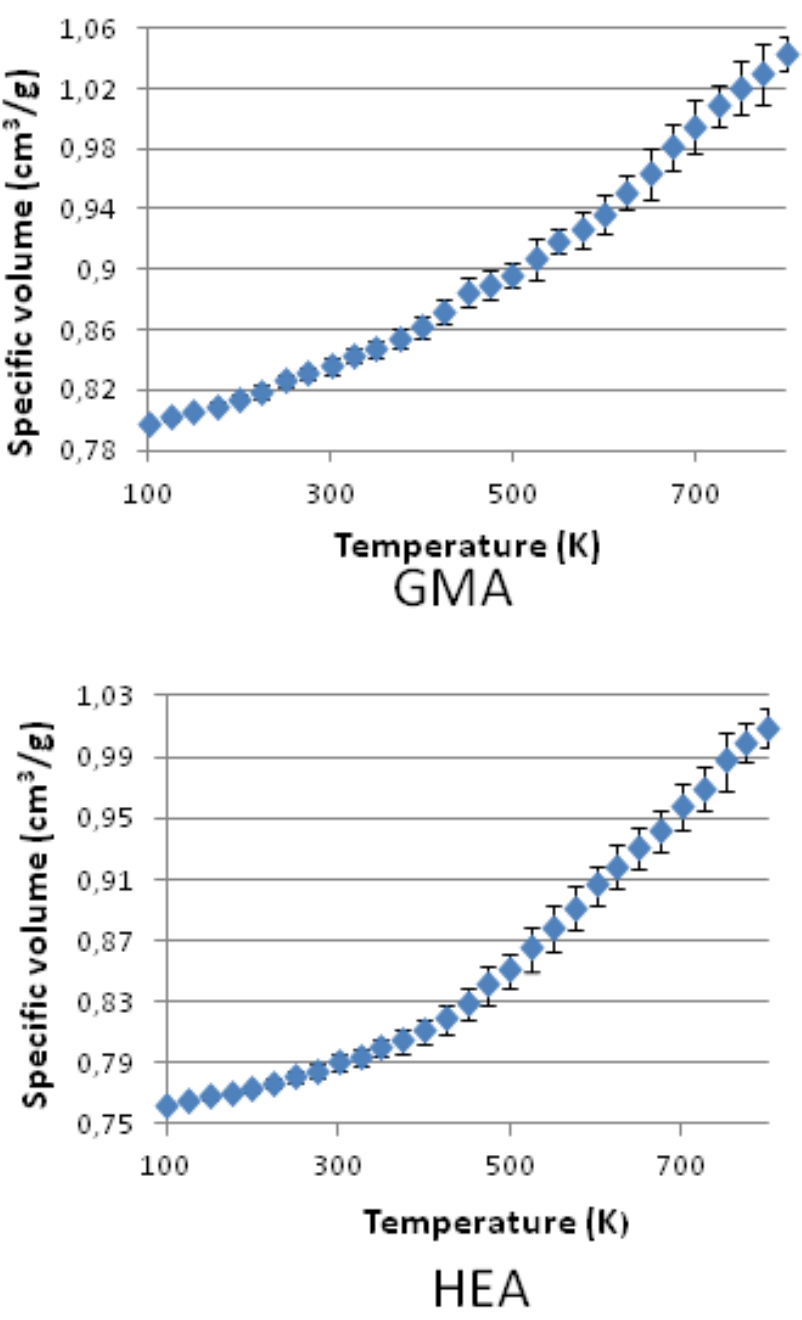

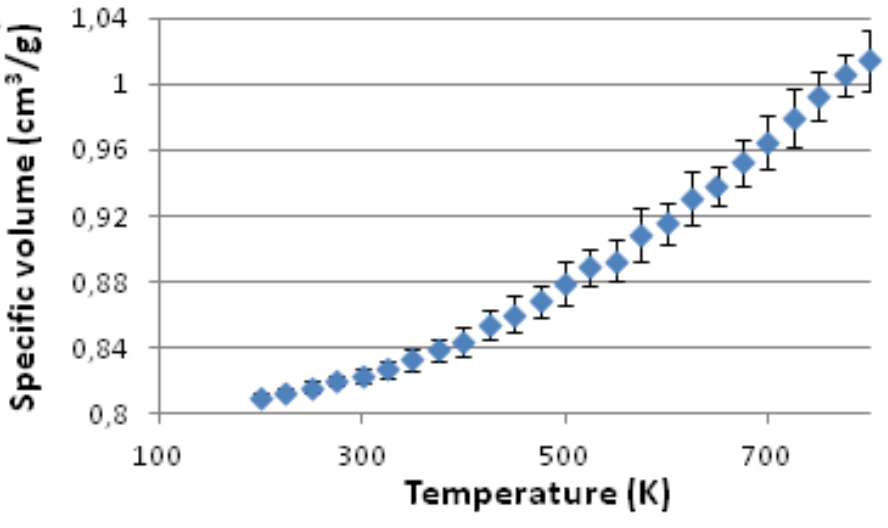

HEMA
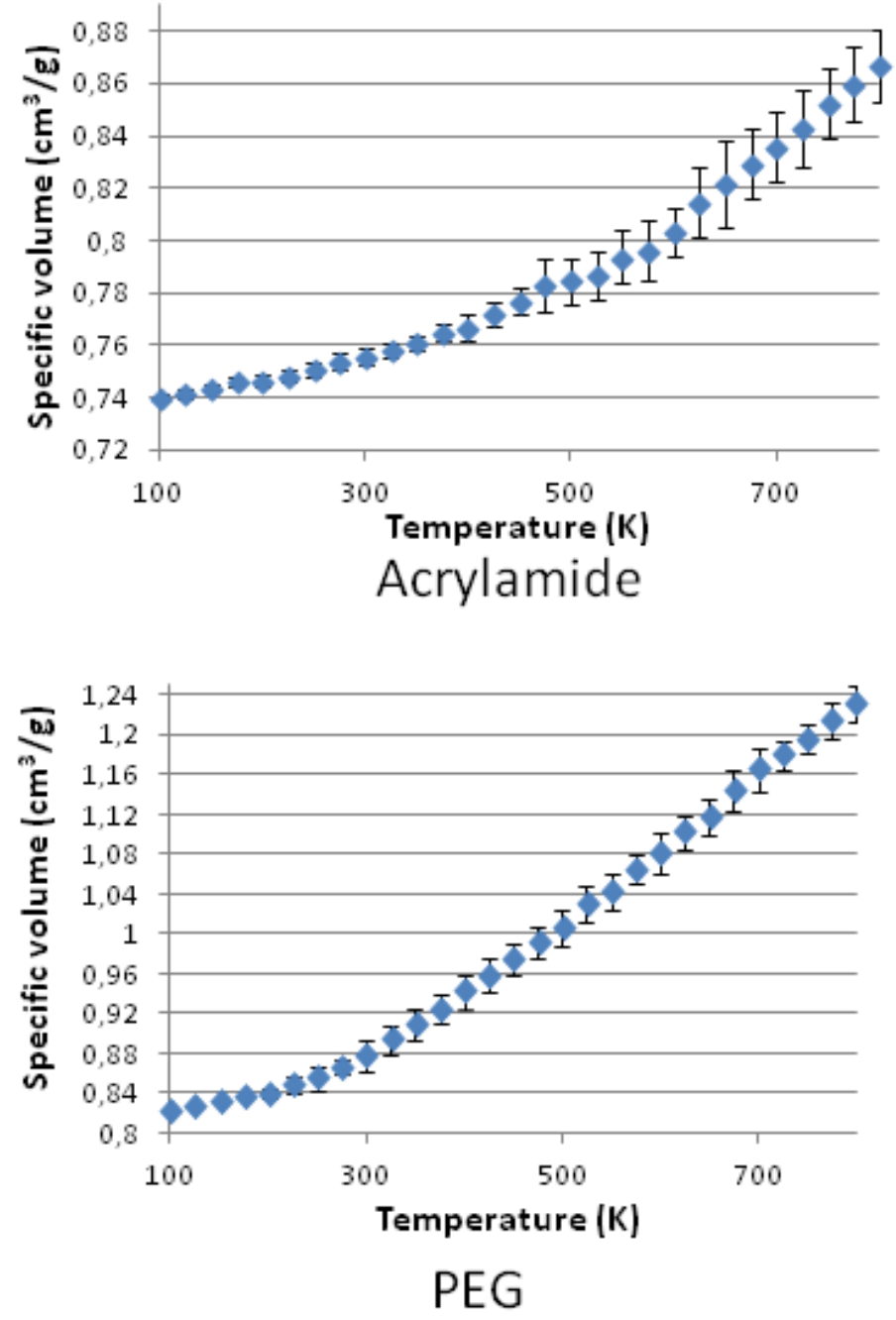

Fig. 3 Specific volume versus temperature for the different dry polymers

Computed values for the glass transition temperature ( $\mathrm{Tg}$ ) are presented along side experimental and group contribution data in Fig. 4. The Tg are calculated from the intersection of the linear regression 
lines obtained on the glassy and amorphous regions. First of all, the computed values always over estimate the experimental one. For HEMA, polyacrylamide, and GMA we noted a difference of respectively 36, 38, and $60 \mathrm{~K}$ whereas the discrepancy for HEA was above $100 \mathrm{~K}$. Concerning the group contribution values, exception made for the HEA, they are closer to the experimental values but always lower (from 8 to $72 \mathrm{~K}$ ). From a global point of view the $\mathrm{Tg}$ from literature were increased in order of polyacrylamide > HEMA > GMA $\approx$ TFEM > HEA > PEG whereas from our result the ranking was polyacrylamide $>\mathrm{TFEM} \approx \mathrm{HEMA} \approx \mathrm{GMA} \approx \mathrm{HEA}>\mathrm{PEG}$.

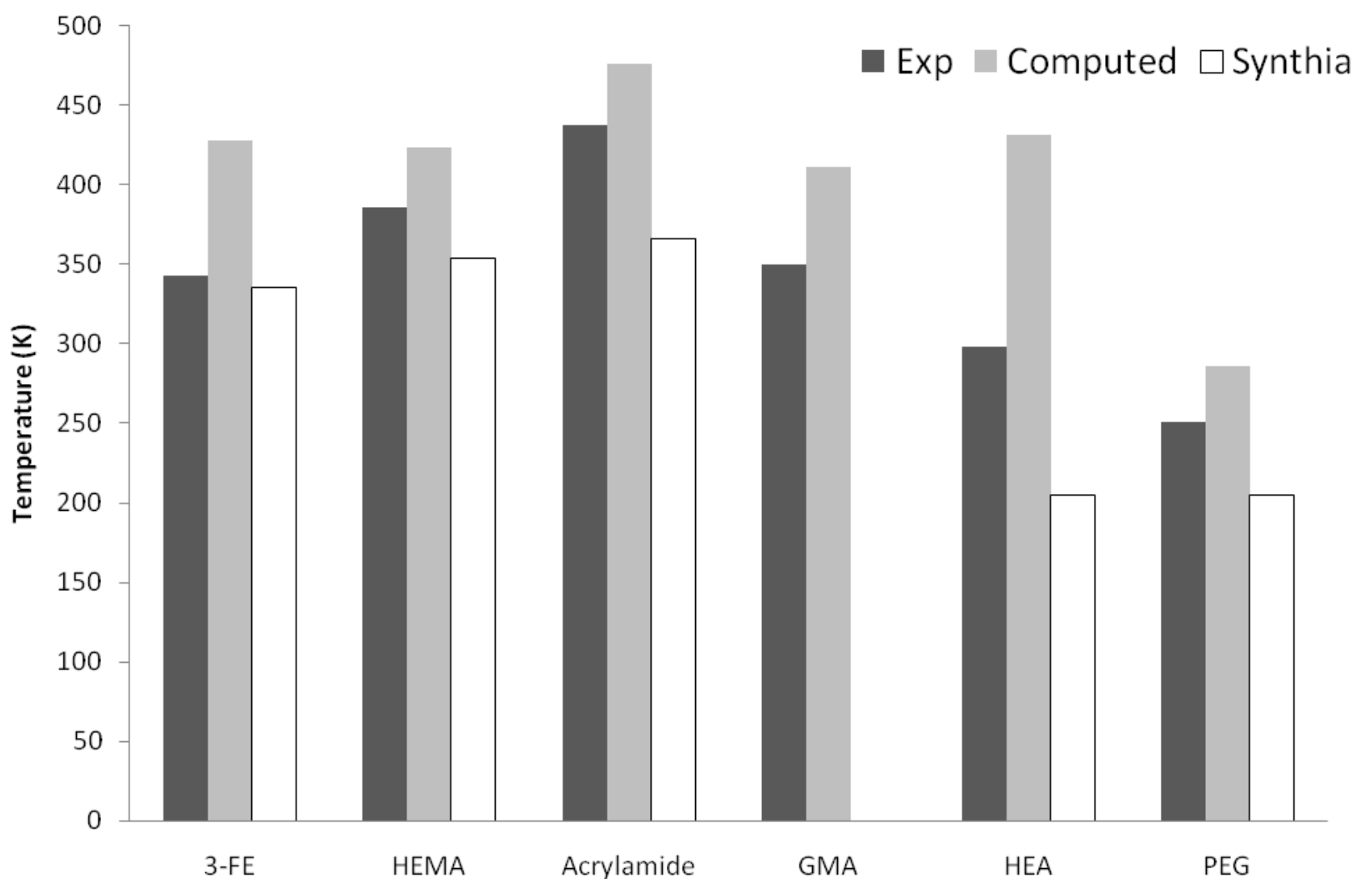

Fig.4 Comparison of calculated glass transition temperature values by molecular dynamic (MD) or group contribution method (Gr.Contr.) with experimental results for TFEM [46]; HEMA [47]; polyacrylamide [48]; GMA [49]; HEA [50]; PEG [51].

The main discrepancies were observed for HEA and TFEM. Even if these values are not aberrant they pose the problem of coherence between the calculated values. In addition to the chemical structures of the repeat units, different parameters can affect the Tg value. Among them we can cite the molecular weight average (Mw), the cooling rate, and the tacticity. From the Fox and Flory equation [33], the variation between $\mathrm{Tg}(\mathrm{Mw}=\infty)$ and $\mathrm{Tg}$ $(\mathrm{Mw})$ is inversely proportional to Mw. Consequently, this effect cannot be the cause of our discrepancy. The experimental data from reference [34] concerning HEA comes from dynamic-mechanical spectroscopy of a dry sample performed with a heating rate of $2{ }^{\circ} \mathrm{C} \min ^{-1}$ from $-125{ }^{\circ} \mathrm{C}$ to $100{ }^{\circ} \mathrm{C}$ at the frequency of $1 \mathrm{~Hz}$. The impact 
of the cooling rate on the comparison between calculated and experimental results was studied by Soldera and Metatla [35] and cannot explain so biga discrepancy. Concerning the tacticity, for HEMA, the Tg varies from 328 to $373 \mathrm{~K}$ for atactic conformations and $382 \mathrm{~K}$ for syndiotactic whereas the Tg was measured at only $308 \mathrm{~K}$ for isotactic configuration [36]. The tacticity was not given in the references, and we do not find information concerning the effect of the tacticity on the Tg of HEA and TFEM but it could be an explanation to the observed discrepancy.

\section{Mechanical properties}

A necessary condition for a comfortable contact lens is to be composed of a material with comparable mechanical properties to the cornea since too much flexibility could lead to residual astigmatism [37]. However, the experimental values of the Young's modulus measured for the cornea are widely dispersed [38]. From indentation measurement the values range from 7.5 to $50 \mathrm{kPa}$ with an average one around $29 \mathrm{kPa}$ whereas from tensile test, the Young's modulus ranges from 0.1 to $11.1 \mathrm{MPa}$, with an average value of $3 \mathrm{MPa}$. Consequently, the prediction of quantitative results is of little interest in this application case, but it's important to obtain reliable qualitative results. Complete elastic constants were evaluated by static method. It's a computationally efficient method to obtain the elastic constants of periodic cell at $0 \mathrm{~K}$ but reputed to provide large scattering values. Considering our periodic cell as a representative volume element of amorphous polymer, the stiffness matrix is expected to be positive definite, symmetric, and isotropic. The stiffness matrixes averaged over the five configurations are given in Table 3. The standard deviation is lower than $1 \mathrm{GPa}$ for $75 \%$ of the elastic constants with only one values higher than $2 \mathrm{GPa}$. In all cases, whatever the polymer is, the dispersion is more important for C11, C22 and C33 components. Taking into account this dispersion, the symmetry is much better after averaging in comparison to what can be seen for only one configuration. Averaging on more configuration could decrease the present dispersion but a significant improvement will be linked to an excessive increase of the computation time cost.

TFEM

\begin{tabular}{c|c|c|c|c|c}
\hline $8 \pm 2$ & $3.7 \pm 0.7$ & $4 \pm 0.7$ & $0.2 \pm 0.5$ & $-0.2 \pm 0.5$ & $-0.3 \pm 0.2$ \\
\hline $3.7 \pm 0.7$ & $7.4 \pm 0.8$ & $3.8 \pm 0.4$ & $0.4 \pm 0.7$ & $-0.1 \pm 0.2$ & $-0.4 \pm 0.3$ \\
\hline $4 \pm 0.7$ & $3.8 \pm 0.4$ & $7.3 \pm 1$ & $0.3 \pm 0.3$ & $-0.3 \pm 0.5$ & $-0.3 \pm 0.2$ \\
\hline $0.1 \pm 0.5$ & $0.4 \pm 0.7$ & $0.3 \pm 0.3$ & $2.1 \pm 0.2$ & $0 \pm 0.2$ & $-0.1 \pm 0.2$ \\
\hline$-0.2 \pm 0.5$ & $-0.1 \pm 0.2$ & $-0.4 \pm 0.5$ & $0 \pm 0.2$ & $2 \pm 0.5$ & $0.2 \pm 0.2$ \\
\hline$-0.3 \pm 0.2$ & $-0.4 \pm 0.3$ & $-0.3 \pm 0.2$ & $-0.1 \pm 0.2$ & $0.2 \pm 0.2$ & $1.9 \pm 0.2$ \\
\hline \multicolumn{6}{|c}{ Acry } \\
\hline $17 \pm 1$ & $8.9 \pm 0.7$ & $8.6 \pm 0.8$ & $0.1 \pm 0.8$ & $0.35 \pm 0.7$ & $-0.1 \pm 0.7$ \\
\hline $9 \pm 0.8$ & $19.3 \pm 2.2$ & $9.6 \pm 1.1$ & $0.3 \pm 1.1$ & $-0.2 \pm 1.3$ & $0.5 \pm 0.7$ \\
\hline $8.6 \pm 1.3$ & $9.6 \pm 0.7$ & $17.3 \pm 0.7$ & $0.6 \pm 0.7$ & $0.5 \pm 0.8$ & $-0.2 \pm 0.7$ \\
\hline $0.1 \pm 0.8$ & $0.3 \pm 1.1$ & $0.6 \pm 0.6$ & $4.9 \pm 0.6$ & $0.1 \pm 0.3$ & $0 \pm 0.4$
\end{tabular}




\begin{tabular}{c|c|c|c|c|c}
$0.5 \pm 0.7$ & $-0.3 \pm 1.3$ & $0.5 \pm 0.3$ & $0.1 \pm 0.3$ & $4.7 \pm 0.8$ & $0.2 \pm 0.2$ \\
\hline$-0.1 \pm 0.8$ & $0.5 \pm 0.7$ & $-0.2 \pm 0.4$ & $0 \pm 0.4$ & $0.2 \pm 0.2$ & $5 \pm 0.4$ \\
\hline \multicolumn{6}{|c}{ GMA } \\
\hline $10 \pm 1$ & $5.3 \pm 0.6$ & $5.3 \pm 0.2$ & $-0.4 \pm 0.1$ & $0.1 \pm 0.4$ & $0 \pm 0.2$ \\
\hline $5.3 \pm 0.6$ & $10.4 \pm 1.8$ & $5.6 \pm 0.5$ & $-0.3 \pm 0.3$ & $0.1 \pm 0.3$ & $0.2 \pm 0.3$ \\
\hline $5.3 \pm 0.2$ & $5.6 \pm 0.5$ & $11 \pm 1.2$ & $-0.2 \pm 0.2$ & $0.2 \pm 0.5$ & $-0.1 \pm 0.3$ \\
\hline$-0.4 \pm 0.2$ & $-0.3 \pm 0.3$ & $-0.2 \pm 0.2$ & $2.3 \pm 0.3$ & $0.1 \pm 0.2$ & $0.1 \pm 0.1$ \\
\hline $0.1 \pm 0.4$ & $0.1 \pm 0.3$ & $0.3 \pm 0.4$ & $0.1 \pm 0.2$ & $2.5 \pm 0.3$ & $-0.2 \pm 0.2$ \\
\hline $0 \pm 0.2$ & $0.2 \pm 0.3$ & $-0.1 \pm 0.3$ & $0.1 \pm 0.1$ & $-0.2 \pm 0.32$ & $2.6 \pm 0.2$ \\
\hline \multicolumn{7}{|c}{ HEA } \\
\hline $13 \pm 1$ & $5.9 \pm 0.5$ & $5.9 \pm 1$ & $0.2 \pm 0.5$ & $0.4 \pm 0.2$ & $-0.1 \pm 0.3$ \\
\hline $5.9 \pm 0.5$ & $13.7 \pm 1$ & $6.5 \pm 1$ & $-0.2 \pm 0.3$ & $0.3 \pm 0.2$ & $-0.2 \pm 0.4$ \\
\hline $5.9 \pm 0.9$ & $6.5 \pm 1.1$ & $13 \pm 0.6$ & $0 \pm 0.3$ & $0.1 \pm 0.3$ & $-0.2 \pm 0.4$ \\
\hline $0.2 \pm 0.5$ & $-0.2 \pm 0.3$ & $0 \pm 0.3$ & $3.2 \pm 0.3$ & $-0.1 \pm 0.3$ & $-0.1 \pm 0.3$ \\
\hline $0.4 \pm 0.2$ & $0.2 \pm 0.3$ & $0.1 \pm 0.3$ & $-0.1 \pm 0.3$ & $3.5 \pm 0.8$ & $0.1 \pm 0.3$ \\
\hline$-0.1 \pm 0.3$ & $-0.2 \pm 0.4$ & $-0.2 \pm 0.4$ & $-0.1 \pm 0.2$ & $0.1 \pm 0.3$ & $3.3 \pm 0.5$ \\
\hline \multicolumn{7}{|c}{ HEMA } \\
\hline $13 \pm 1$ & $6.6 \pm 0.4$ & $6.7 \pm 0.4$ & $0.5 \pm 0.2$ & $0.2 \pm 0.8$ & $0.1 \pm 0.1$ \\
\hline $6.6 \pm 0.4$ & $13.9 \pm 1.1$ & $6.4 \pm 0.9$ & $-0.1 \pm 0.3$ & $0.1 \pm 0.3$ & $0.2 \pm 0.5$ \\
\hline $6.7 \pm 0.5$ & $6.4 \pm 0.9$ & $13.5 \pm 1.5$ & $0.3 \pm 0.4$ & $-0.1 \pm 0.3$ & $0.4 \pm 0.4$ \\
\hline $0.5 \pm 0.2$ & $-0.1 \pm 0.3$ & $0.3 \pm 0.4$ & $3.1 \pm 0.2$ & $0.1 \pm 0.2$ & $0.1 \pm 0.4$ \\
\hline $0.2 \pm 0.8$ & $0.1 \pm 0.3$ & $-0.1 \pm 0.3$ & $0.1 \pm 0.2$ & $3.3 \pm 0.4$ & $0 \pm 0.1$ \\
\hline $0.1 \pm 0.1$ & $0.2 \pm 0.6$ & $0.4 \pm 0.4$ & $0.1 \pm 0.4$ & $0 \pm 0.1$ & $3.3 \pm 0.4$ \\
\hline \multicolumn{7}{|c}{ PEG } \\
\hline $12 \pm 2$ & $6.28 \pm 1$ & $6.7 \pm 0.8$ & $0.2 \pm 0.4$ & $0.1 \pm 0.8$ & $-0.1 \pm 0.6$ \\
\hline $6.8 \pm 1$ & $11.8 \pm 1.9$ & $7.1 \pm 1.4$ & $0.4 \pm 0.4$ & $-0.6 \pm 0.6$ & $-0.2 \pm 0.6$ \\
\hline $6.7 \pm 0.7$ & $7.1 \pm 1.3$ & $11.1 \pm 1.5$ & $0.3 \pm 0.4$ & $0.1 \pm 0.5$ & $-0.5 \pm 0.5$ \\
\hline $0.3 \pm 0.4$ & $0.4 \pm 0.4$ & $0.3 \pm 0.4$ & $2.6 \pm 0.4$ & $0.1 \pm 0.2$ & $-0.2 \pm 0.4$ \\
\hline $0.1 \pm 0.8$ & $-0.6 \pm 0.6$ & $0.1 \pm 0.5$ & $0.1 \pm 0.2$ & $2.2 \pm 0.4$ & $-0.1 \pm 0.3$ \\
\hline$-0.1 \pm 0.6$ & $-0 . \pm 0.6$ & $-0.5 \pm 0.5$ & $-0.2 \pm 0.4$ & $-0.1 \pm 0.1$ & $2.3 \pm 0.4$
\end{tabular}

Table 3 Stiffness matrix with $\mathrm{Cij}$ in GPa averaged on the five configurations for the different dry homopolymers

The elastic parameters calculated from the elastic constants (EC) provide us with the different moduli for a $0 \mathrm{~K}$ temperature (Fig. 5). Consequently, we can expect that the calculated values over estimate the experimental ones. Unfortunately, the mechanical properties are not abundant for these polymers in their dry state. Consequently, we compared them with the data averaged on the results predicted by group contribution techniques. These latter values, obtained for $298 \mathrm{~K}$, are $40 \%$ lower than those evaluated at $0 \mathrm{~K}$ from EC. The highest bulk modulus is obtained for polyacrylamide whereas (HEMA, HEA) and TFEM are around 18 and $50 \%$ lower respectively for both methods. Concerning GMA, no data was available from group contribution whereas the modulus calculated at $0 \mathrm{~K}$ is $4 \mathrm{GPa}$ lower than for polyacrylamide. It's worth noting that the bulk moduli at $0 \mathrm{~K}$ extracted from the fitting of the energy versus volume curve with a second order polynomial regression model differ by less than 1 
$\mathrm{GPa}$ with those calculated from the elastic constants. In general, the Young's modulus (E) is lower than the bulk modulus for polymers. This trend was observed for group contribution and EC results but for acrylamide. Group contribution method gave a relatively close E for HEA, HEMA and polyacrylamide around 4.5 GPa whereas for TFEM the predicted values is $2.9 \mathrm{GPa}$. The discrepancy between TFEM and HEA/ HEMA is well reproduced from EC results but with an overestimation from 2.3 to $4.4 \mathrm{GPa}$ respectively for TFEM and HEA. Once again, this discrepancy can be partially explained by the difference of temperature. For Instance, the Young's modulus of PCV rises from 3 to $7.5 \mathrm{GPa}$ with a decrease of the temperature from 298 to $75 \mathrm{~K}$ [36]. This over estimation was confirmed by the experimental results found for HEA. Although, the storage and the young's modulus are conceptually different, comparison can be made as far as the damping is insignificant. We can assume that this assumption holds for HEA at temperature well below the glass transition temperature. The storage modulus of HEA crosslinked with $1 \%$ weight of ethylene glycol dimethacrylate was indeed evaluated from dynamic mechanical analysis at $3000 \mathrm{MPa}$ at $240 \mathrm{~K}(\mathrm{Tg}(\mathrm{HEA})=300 \mathrm{~K})$ [39], in agreement with the group contribution predicted value. However, the result for polyacrylamide seems abnormally high even for $0 \mathrm{GPa}$.
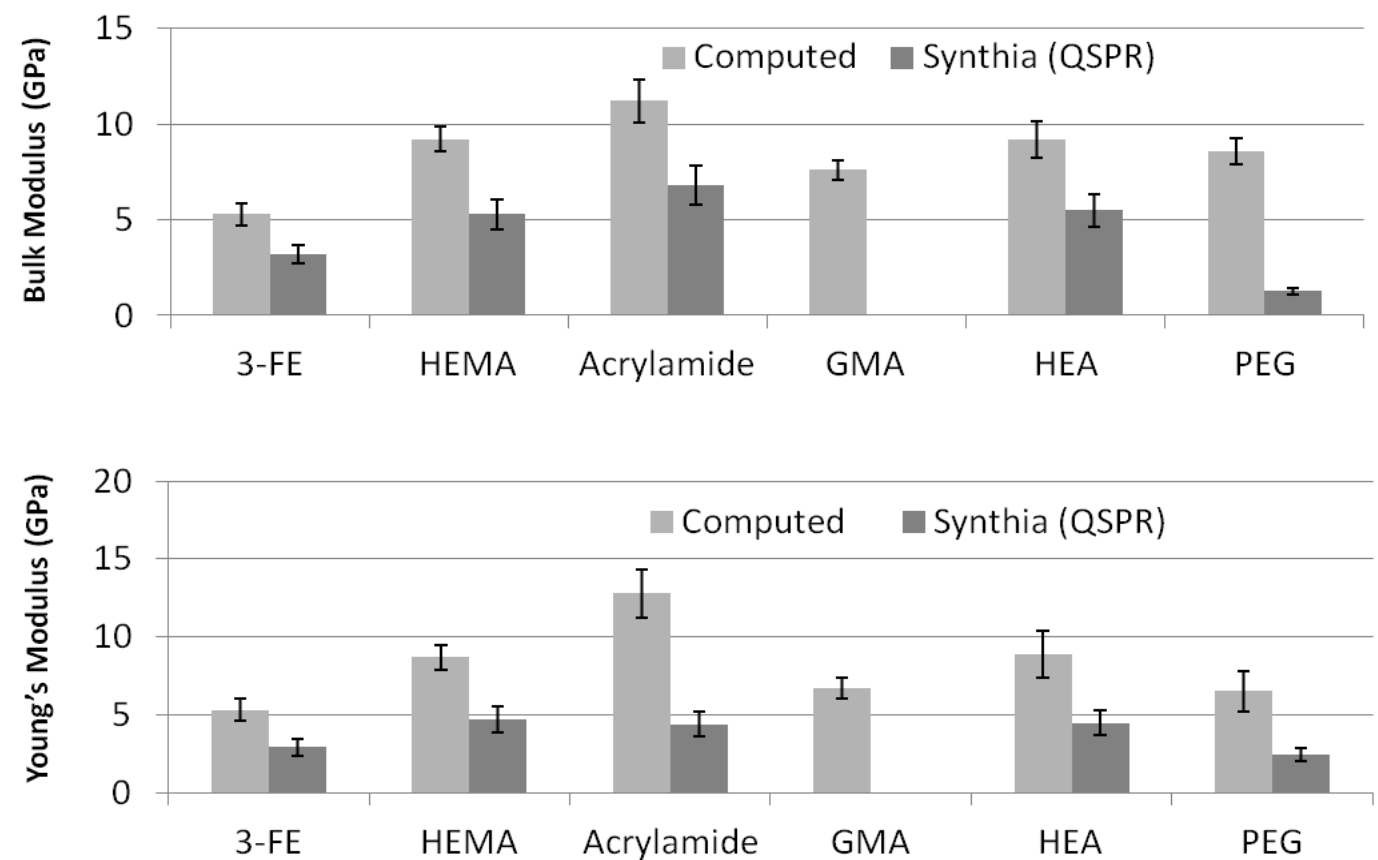

Fig.5 Comparison of bulk and Young's moduli obtained from calculated elastic constants (MM) and group contribution method (Gr. Contr.)

\section{Solubility parameter}

The solubility parameter will give us important information concerning the compatibility between 
the different homopolymers. This property is crucial for the formulation of multicomponent material such as that used in contact lens.Three patents [4, 40,41] dealing with the utilityof this parameter on the design of contact lens materials are given as examples. Moreover, from the semi IPN structure obtained from polymerized MMA-monomer and PMMA-polymer, Vuorinen et al. [42] stated that the formation of a IPN bond is linked to the difference between the solubility parameter of the polymer and the monomer. The solubility parameters calculated from the five configurations equilibrated at $300 \mathrm{~K}$ are presented, for the different homopolymers, in Fig. 6 with the corresponding group contribution values.

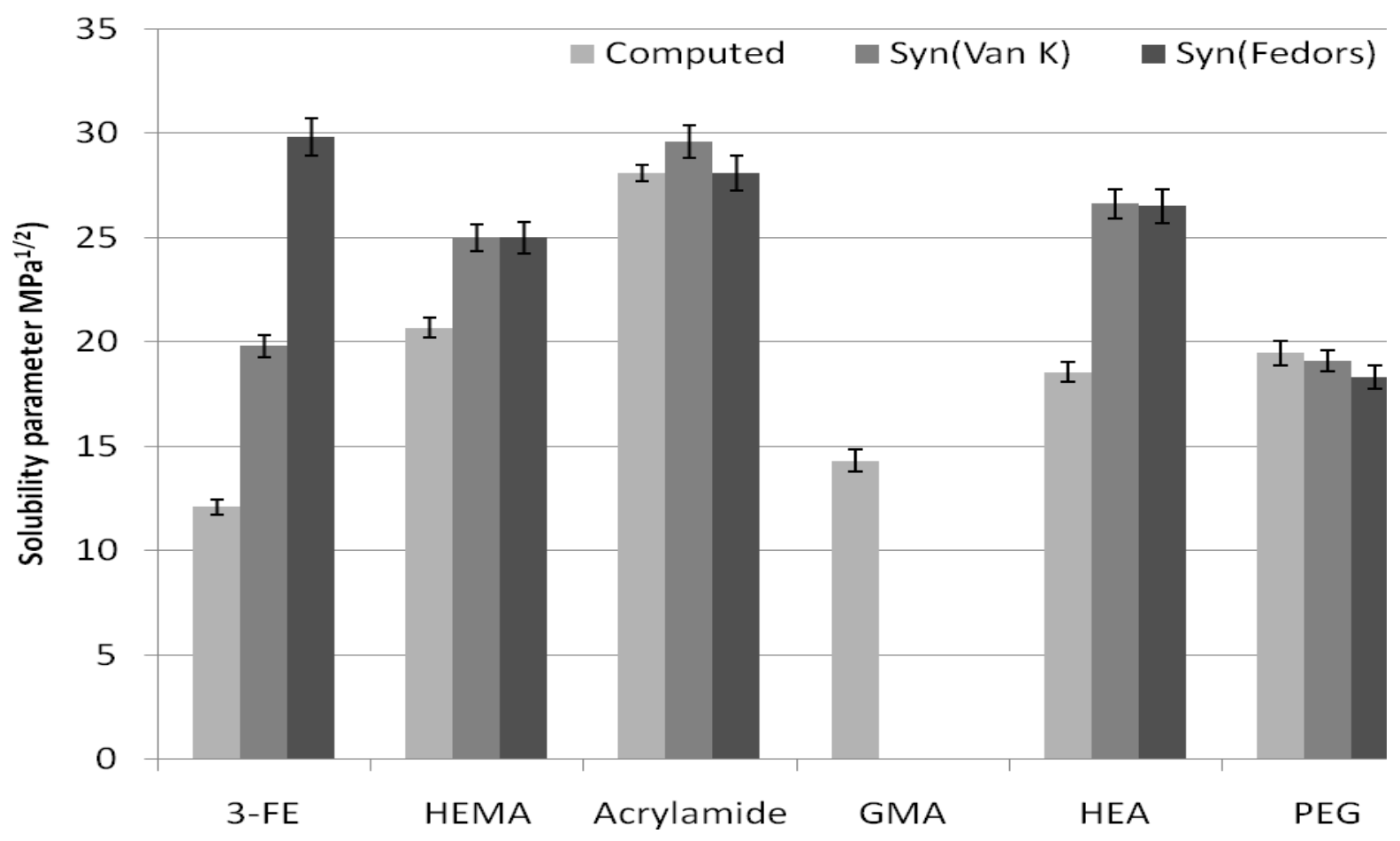

Fig.6 Comparison of the solubility parameters calculated on the configurations at 300K by molecular dynamic(MD) and group contribution methods (Gr. Contr.) byVanKrevelen (1) and Fedors (2).

Two different group contribution methods were available and generally the computed values from molecular dynamics are lower with the exception of the PEG value. For TFEM, we note important discrepancy in the values calculated from the two group contribution methods and the computed value. However, in comparison with the solubility parameter given for themonomer molecule $(\delta(\mathrm{TFEM})=$ $15.9 \mathrm{MPa}^{0.5}$ [43]) the discrepancy is also important. Nevertheless, the solubility parameter of TFEM remains the weakest value independently of the method in comparison with the other polymers. Moreover, the ranking obtained from both computed and literature values is similar polyacrylamide (30.8 $\left.\mathrm{MPa}^{0.5}[44]\right)>\operatorname{HEMA}\left(29.6 \mathrm{MPa}^{0.5}[45]\right)>\operatorname{GMA}\left(20.5 \mathrm{MPa}^{0.5}[32]\right)>\operatorname{TFEM}\left(15.9 \mathrm{MPa}^{0.5}\right.$ [43]). For GMA 
the solubility parameter was calculated using the atomic increments approach proposed by Askadskii, whereas concerning HEMA, the solubility parameter, as usually done for polymer, was matched to that of the solvent with the maximum solvating power, the methanol in this case.

\section{Conclusions}

Classical molecular dynamics simulations were carried out on five hydrophilic and one hydrophobic polymers. The models were composed of one chain of atactic chain containing 100 repeat units. The method developed by Metatla and Soldera was used to generate five equilibrated configurations of amorphous cell for each polymer. The reliability of the results averaged on the five configurations was considered whereas COMPASS force field was used to calculate static properties of interest for contact lens materials development. Densities obtained at $0 \mathrm{~K}$ by extrapolation of the linear regression obtained in the glassy area of the specific volume versus temperature curves and by the fitting of the energy versus volume curve with a second order polynomial regression model were consistent. At $300 \mathrm{~K}$, the result obtained for GMA is abnormally far from the experimental data. For density and solubility parameter our rankings were similar to those obtained from experimental data collected in literature. It is not the case for the glass transition temperature. The discrepancies observed between the experimental and calculated values are higher than expected for TFEM, GMA, and HEA. Concerning the elastic properties, the elastic constants averaged on the five configurations allow a stiffness matrix with characteristics in agreement with the expectations for an amorphous cell model. The bulk moduli calculated by two different methods, the elastic constants and the energy versus volume curve, are consistent. The Young's moduli calculated from the elastic constants were notalways lower than the bulk modulus as generally expected. As a conclusion, COMPASS force field allows us to obtain reliable results as far as the ranking of polymers are concerned. Calculations onbinary andternarycompounds can now be undergone. Comparison with experimental results will require well definedexperimental structures in the aim to compare similar polymeric systems.

\section{References}

1. Barr, J.T.(2005).Contact Lens Spectrum's annual report of major corporate and product developments and events in the contact lens industry in 2004, as well as predictions for 2005. Contact Lens Spectr (January2005), 1-6

2. Contact Lens Market (2015) Gas permeable contact lens and soft contact lens — global industry analysis, size, share, growth, trends and forecast, $2013-2019$.

3. GunsterenWvan, MarkA(1998)Validation of molecular dynamics simulation. J Chem Phys 108:61096116 
4. Chiessi E, Cavalieri F, Paradossi G (2005) Supercooled water in PVA matrixes. II. A molecular dynamics simulation study and comparison with QENS results. J Phys Chem B109:8091-8096

5. Chiesi E, Cavalieri F, Paradossi G (2007) Water and polymer dynamics in chemically cross-linked hydrogels of poly(vinylalcohol): a molecular dynamics simulation study. J Phys Chem B111:2820-2827

6. Zhang H, Wang H, Xu G, Yuan S (2013) A molecular dynamics simulation of N-(fluorenyl-9methoxycarbonyl)-dipeptides supramolecular hydrogel. 417: 217-223

7. Salahshoor H, Rahbar N (2014) Multi-scale mechanical and transport properties of a hydrogel. J Mech Behav Biomed 37:299-306

8. Escobedo FA, de Pablo JJ (1999) Molecular simulation of polymeric networks and gels : phase behavior and swelling. Phys Rep 318:85-112

9. Saramak J, Halagan K, Kozanecki M, Polanowski P (2014) Computational studies of intermolecular interactions in aqueous solutions of poly(vinylmethylether). J Mol Model 20(2529):2-9

10. Wu C (2010) Cooperative behavior of poly(vinylalcohol) and water as revealed by molecular dynamics simulations. Polymer 51:4452-4460

11. Walter J, Ermatchkov VJ, Hasse H (2010) Molecular dynamics and experimental study of conformation change of poly(Nisopropylacrylamide) hydrogels in water. Fluid Phase Equilibr 296:164172

12. Walter J, Sehrt J, Vrabec J, Hasse H (2012) Molecular dynamics and experimental study of conformation change of poly(Nisopropylacrylamide) - hydrogels in mixtures of water and methanol. J Phys Chem B116:5251-5259

13. Karlsson GE, Gedde UW, Hedenqvist MS (2004) Molecular dynamics simulation of oxygen diffusion in dry and water-containing poly(vinyl alcohol). Polymer 45:3893-3900

14. Bermejo JS, Ugarte CM (2008) Influence of water content on structure and mobility of polyvinylalcohol: a molecular dynamics simulation. J Chem Phys 129:1549071-10

15. Zhang QG, Liu QL, Chen Y, Wu JY, Zhu AM (2009) Microstructure dependent diffusion of waterethanolin swollen poly(vinylalcohol) : a molecular dynamics simulation study. Chem Eng Sci 64:334340

16. Müller-Plathe F (1998) Diffusion of water in swollen poly(vinyl alcohol) membranes studied by molecular dynamics simulation. J Memb Sci 141:147-154

17. Lee SG, Koh W, Brunello GF, Choi JI, Bucknall DG, Jang SS (2012) Effect of monomeric sequence on transport properties of D-glucose and ascorbic acid in poly(VP-co-HEMA) hydrogels with various water contents: molecular dynamics simulation approach. Theor Chem Acc 131:2-16 
18. Sun D, Zhou J (2012) Effect of water content on microstructures and oxygen permeation in PSiMAIPN-PMPC hydrogel: a molecular simulation study. Chem Eng Sci78:236-245

19. Pozuelo J, Compañ V, González-Méijome JM, González M, Mollá S (2014) Oxygen and ionic transport in hydrogel and siliconehydrogel contact lens materials: an experimental and theoretical study. J Membr Sci 452:62-72

20. Jang SS, Goddard WAIII (2007) Mechanical and transport properties of the poly(ethyleneoxide)poly(acrylic acid) double network hydrogel from molecular dynamic simulations. J Phys Chem B111:1729-1737

21. Lee SG, Brunello GF, Jang SS, Bucknall DG (2009) Molecular dynamics simulation study of P(VPco-HEMA) hydrogels : effect of water content on equilibrium structures and mechanical properties. Biomaterials 30(30):6130-6141

22. Noorahan A, Choi P (2013) Thermodynamic properties of poly(vinylalcohol) with different tacticities estimated from molecular dynamics simulation. Polymer 54:4212-4219

23. Kindt-Larsen T, Heaton JC, Rastrelli EC (1988). Molding hydrophilic(meth)acrylate ester and water displacable boric acid ester diluent. Patent US 4889664A

24. Lira M, Santos L, Azeredo J, Yebra-Pimente IE, Oliveira MECDR (2008) Comparative study of silicone-hydrogel contact lenses surfaces before and after wear using atomic force microscopy. J Biomed Mater Res Part B,Appl Biomater 85(2):361-367

25. Metatla N, Soldera A (2006) Computation of densities, bulk moduli and glass transition temperatures of vinylic polymers from atomistic simulation. Mol Simul32(14):1187-1193

26. Theodorou D, Suter U (1985) Detailed molecular structure of a vinyl polymer glass. Macromolecules 1467:1467-1478

27. Sun H (1998) COMPASS: an ab initio force-field optimized for condensed-phase applications overview with details on alkane and benzene compounds. J Phys Chem B102(38):7338-7364

28. Van Krevelen DW, Te Nijenhuis K (2009). Properties of polymers: their correlation with chemical structure; their numerical estimation andprediction from additive group contributions. Elsevier, Amsterdam

29. Bicerano J (2002) Prediction of Polymer Properties, 3rd edn. Dekker, NewYork

30. VanDijk MA, Wakker A (1997) Concepts in polymer thermodynamics, volume 2. Technomic, Lancaster

31. Ding Y, Kisliuk A, Sokolov AP (2004) When does a molecule become a polymer? Macromolecules 37:161-166 
32. Zdyrko B, Swaminatha IK, Luzinov I (2006) Macromolecular anchoring layers for polymer grafting: comparative study. Polymer 47(1):272-279

33. Fox TG, Flory PJ (1950) Second order transition temperatures and related properties of polystyrene. I. Influence of molecular weight. J Appl Phys 21:581-591

34. Gallego FG, Salmeron SM, Gomez Ribelles JL, Romero Colomer FJ, Monleon PM (2007) Nanodomain in a hydrophilic-hydrophobic IPN based on poly(2-hydroxyethyl acrylate) and poly(ethyl acrylate),(2007). Eur Polym J 43(8):3163-3145

35. Soldera A, Metatla N (2006) Glass transition of polymers: Atomistic simulation versus experiments. Phys Rev E 74(6):061803

36. Mark JE (1999) Polymer data handbook. Oxford University Press

37. French K (2005) Contact lens material properties part 2 : mechanical behaviour and modulus. Optician 230:29-34

38. McKee C, Last J (2011) Indentation versus tensile measurements of Young's modulus for soft biological tissues. Eng Part B17:155-164

39. Gomez Tejedor JA, Rodriguez AT, Gomez Ribelles JL, Polizos G, Pissis P (2007) Poly(ethyl methacrylate-co-hydroxyethyl acrylate) random co-polymers: dielectric and dynamic-mechanical characterization. J Non Crys 353:276-285

40. Kindt-Larsen T, Molock FF, Rossignol H, Sorensen JE, Steenstrup FR, Wolff P (2005) Low polydispersity poly-hema compositions. Patent US6846892B2

41. Vanderlaan DG, Petisce JR, Azaam A, Mccabe KP, Rathore O (2004) Diluents for forming clear, wettable silicone hydrogel articles. Patent CA2518108C

42. Vuorinen A-M, Dyer SR, Lassila LVJ, Vallittu PK (2008) Effect of rigid rod polymer filler on mechanical properties of poly-methyl methacrylate denture base material. Dent Mater: Off Publ Acad Dent Mater 24(5):708-713

43. Koike Y (2000) Refractive index distribution type optical fibre and method of manufacturings aid optical fibre. Patent EP0566744B1

44. Lopatin VV, Askadskii A, Peregudov AS, Vasil'ev VG (2005) Structure and relaxation properties of medical-purposed polyacrylamide gels. J Appl Polym Sci 96(4):1043-1058

45. Okay O, Gürün Ç (1992) Synthesis and formation mechanism of porous 2-hydroxyethyl methacrylate-ethyleneglycoldimethacrylate copolymer beads. J Appl Polym Sci 46:401-410

46. Higgenbotham-Bertolucci P (2001) Creep and stress relaxationin methacrylate polymers: two mechanisms of relaxation behavior across the glass transition region. Polym Eng 41(5) 
47. Meakin JR, Hukins DWL, Imrie CT, Aspden RM (2003) Thermal analysis of poly(2-hydroxyethyl methacrylate) (pHEMA) hydrogels. J Mater Sci Mater Med 14(1):9-15

48. Cheremisinoff NP (1989) Handbook of polymer science and technology (vol.4). CRC Press, BocaRaton

49. Park CK, Ha CS, Lee JK, Cho WJ (1993) Miscibility of polychloroprene/polyglycidylmethacrylate blends and effects of poly(chloroprene-co-glycidylmethacrylate) addition. Polymer (Korean) 18(1):78-85 50. Ferrer GG, Sánchez MS, Ribelles JLG, Colomer FJR, Pradas MM (2007) Nanodomains in a hydrophilic-hydrophobic IPN based on poly(2-hydroxyethylacrylate) and poly(ethyl acrylate). Eur Polym J 43(8):3136-3145

51. Feng L, Bian X, Li G, Chen Z, Cui Y, Chen X (2013) Determination of ultra-low glass transition via differential scanning calorimetry. Polym Test 32(8):1368-1372 